Pacific Journal of Mathematics

ON CERTAIN POINT-COUNTABLE COVERS

anis K. Burke and ERnest A. Michael 


\section{ON CERTAIN POINT-COUNTABLE COVERS}

\section{Dennis Burke and Ernest Michael}

In recent years, there have been a number of results about spaces with a point-countable cover satisfying various assumptions. In this paper, these results are generalized and unified by showing that the assumptions used can be significantly weakened. We are mostly concerned with consequences of the following condition: There is a point-countable cover $\mathscr{P}$ of the space $X$ such that, if $x, y \in X$ with $x \neq y$, then $\mathscr{P}$ has a finite subcollection $\mathscr{F}$ such that $x \in(\cup \mathscr{F})^{\circ}$ and $y \notin \cup \mathscr{F}$.

1. Introduction. The purpose of this note is to make some contributions to the study of point-countable ${ }^{1}$ covers. We will consider topological spaces $X$ with the following properties.

(1.1) $X$ has a point-countable base.

(1.2) $X$ has a point-countable open cover $\mathscr{P}$ which separates points (i.e., if $x, y \in X$ with $x \neq y$, then there is a $P \in \mathscr{P}$ such that $x \in P$ and $y \notin P$ ).

(1.3) $X$ has a point-countable cover $\mathscr{P}$ such that, if $x \in W$ with $W$ open in $X$, then there is a finite subcollection $\mathscr{F}$ of $\mathscr{P}$ such that $x \in(\cup \mathscr{F})^{\circ}, \cup \mathscr{F} \subset W$, and $x \in \cap \mathscr{F}$.

(1.4) Same as (1.3), but without requiring $x \in \cap \mathscr{F}$.

(1.5) $X$ has a point-countable cover $\mathscr{P}$ such that if $x, y \in X$ with $x \neq y$, there is a finite subcollection $\mathscr{F}$ of $\mathscr{P}$ such that $x \in(\cup \mathscr{F})^{\circ}$ and $y \notin \cup \mathscr{F}$.

We have the following implications for a space $X$ :

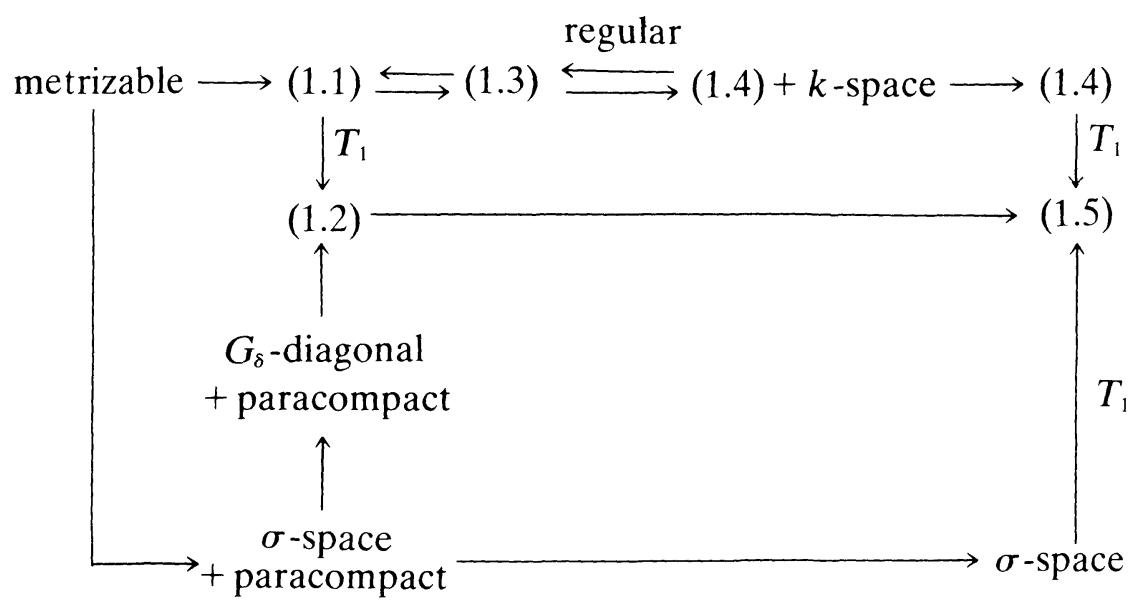

'A collection $\mathscr{P}$ of subsets of $X$ is point-countable if every $x \in X$ is in at most countably many $P \in \mathscr{P}$. 
The implications $\quad(1.1) \rightarrow(1.3) \rightarrow(1.4) \stackrel{T_{1}}{\rightarrow}(1.5)$ and $(1.1) \stackrel{T_{1}}{\rightarrow}(1.2)$ $\rightarrow(1.5)$ are clear. The Nagata-Smirnov or the Bing metrization theorem implies that metrizable spaces satisfy (1.1). That $(1.3) \rightarrow(1.1)$ was proved by the authors in [6]; hence (1.3) implies that $X$ is first-countable ${ }^{2}$ and thus a $k$-space. That (1.4) implies (1.3) if $X$ is a regular $k$-space will be established in Theorem 6.2. J. Nagata observed in [18] that paracompact $^{3}$ spaces with a $G_{\delta}$-diagonal satisfy (1.2). All Hausdorff $\sigma$-spaces (see $\$ 5$ ) have a $G_{\delta}$-diagonal because their closed subsets are $G_{\delta}$ 's and the product of two $\sigma$-spaces is a $\sigma$-space. Finally, Theorem 5.2 will show that every $T_{1} \sigma$-space satisfies $(1.5)^{4}$.

Spaces satisfying (1.1) have been characterized as the open $s$-images ${ }^{5}$ of metric spaces by V. I. Ponomarev [21] and S. Hanai [10], as the bi-quotient ${ }^{6} s$-images of metric spaces by V. V. Filippov [9], and (if Hausdorff) as the compact-covering ${ }^{7}$ open $s$-images of metric spaces by K. Nagami and the second author [14]. A simpler proof of Filippov's main result (that (1.1) is preserved by bi-quotient $s$-maps) was given by the authors in [6]; the crucial step in that proof was to show that $(1.3) \rightarrow(1.1)$.

In a different direction, it has been shown that, in certain kinds of spaces, (1.1) or even (1.2) is equivalent to metrizability. Thus A. S. Miščenko [15] and M. E. Rudin (unpublished ${ }^{8}$ ) showed that (1.1) is equivalent to metrizability in compact Hausdorff spaces, and V. V. Filippov [8] extended this to paracompact $p$-spaces (= paracompact $M$-spaces). In [18], J. Nagata then showed that Filippov's theorem (and thus also the Miščenko-Rudin theorem) remains valid with (1.1) weakened to (1.2), and the second author showed in [11] that a regular strong $\Sigma$-space (see $\$ 5$ ) satisfying (1.2) is a $\sigma$-space ${ }^{9}$; we will show in Theorems 3.1, 4.2, and 5.2 that, in all these results, (1.2) can be weakened to $(1.5)$.

2 This is easily verified directly.

${ }^{3}$ Paracompact can be weakened to meta-Lindelöf (i.e., every open cover has a point-countable open refinement).

${ }^{4}$ By contrast, it was shown in [5, Example 2.4.5] (see also [4]) that a $\sigma$-space - in fact, a locally compact Moore space - need not satisfy (1.2). This implies that, even in a locally compact Moore space, (1.5) need not imply (1.2), in striking contrast to the fact that (1.4) implies (1.1) in any regular $k$-space.

'A map $f: X \rightarrow Y$ is an $s$-map if every $f^{-1}(y)$ has a countable base.

${ }^{6}$ A map $f: X \rightarrow Y$ is bi-quotient if, whenever $y \in Y$ and $U$ is a cover of $f^{-1}(y)$ by open subsets of $X$, then $y \in(f(\cup \mathscr{F}))^{\circ}$ for some finite $\mathscr{F} \subset \mathcal{U}$.

' A map $f: X \rightarrow Y$ is compact-covering if every compact $K \subset Y$ is the image of some compact $C \subset X$.

${ }^{8}$ A modification of Rudin's proof (which also works for countably compact spaces) is given in [7, Proposition 2.1].

9 A more direct proof, which does not need regularity, was subsequently obtained by T. Shiraki [22] and, independently, by F. G. Slaughter, Jr. (unpublished). 
Finally, a result of G. Aquaro [1] implies that the results involving (1.2) which were stated in the previous paragraph remain valid with compact weakened to countably compact (and thus with paracompact $M$-space weakened to Hausdorff $M$-space, and with strong $\Sigma$-space weakened to $\Sigma$-space). We will show in $\$ 7$ that this also remains true if (1.2) is weakened to (1.5), provided the space is a regular $k$-space or a regular $c$-space (see $\$ 2$ for definition). Whether some such additional hypothesis is really necessary, however, remains an open question.

In $\S 8$ we include some remarks on two modifications of (1.5). One of these modifications is formally weaker than (1.5) and the other is formally stronger; it is shown, however, that the two modifications are equivalent to (1.5) whenever the space is a $c$-space.

Section 2 is devoted to preliminary lemmas, while $\$ \S 3-8$ contain our main results. Theorem 3.1 is used in the proofs of Theorems 4.2, 6.2 and 7.2, and Theorem 4.2 is used to prove Theorem 5.2.

We adopt the convention that regular spaces are $T_{1}$ and paracompact spaces are Hausdorff; however, no other separation axioms are assumed unless otherwise stated.

2. Some lemmas. We record here five lemmas which are needed in the sequel. Lemma 2.1 is applied in the proof of Theorem 3.1 , and the other lemmas in later results.

Lemma 2.1 (A. S. Miščenko [15]). If $\mathscr{P}$ is a point-countable cover of a set $X$, then every $A \subset X$ has only countably many minimal finite covers by elements of $\mathscr{P}$.

Our next lemma, which was proved by the authors in [6, Remark 4.1], reduces to Lemma 2.1 when $X$ is a discrete space. Recall that a space $X$ is a $c$-space ( = space determined by countable subsets $=$ space of countable tightness) if a set $A \subset X$ is closed whenever $\bar{C} \subset A$ for every countable $C \subset A$. Every first-countable space (more generally, every sequential space) and every hereditarily separable space is a $c$ space. See $[12, \S 8]$ for more details.

Lemma 2.2 [6]. Suppose $X$ is a $c$-space and $A \subset X$. If $\mathscr{P}$ is any point-countable collection of subsets of $X$, then there are at most countably many minimal finite subcollections $\mathscr{F} \subset \mathscr{P}$ such that $A \subset(\cup \mathscr{F})^{\circ}$. (Minimal means that $A \not \subset(\cup \mathscr{E})^{\circ}$ if $\mathscr{E} \subset \mathscr{F}$ and $\left.\mathscr{E} \neq \mathscr{F}\right)$.

It should be remarked that the $c$-space hypothesis cannot be omitted from Lemma 2.2, even when $X$ is compact. Consider, for example, the space of ordinals $X=\left[0, \omega_{1}\right]$, with the usual order topology. Let $\mathscr{P}$ 
consist of $\left\{\omega_{1}\right\}$ and all open intervals $\left(\alpha, \omega_{1}\right)$ with $\alpha<\omega_{1}$. Then $\mathscr{P}$ is point-countable, but the conclusion of Lemma 2.2 fails for $A=\left\{\omega_{1}\right\}$.

The following two lemmas will sometimes be useful when applying Lemma 2.2.

Lemma 2.3. Suppose $f: X \rightarrow Y$ is continuous and closed, with $X$ regular. If $Y$ and all $f^{-1}(y)$ are first-countable, so is $X$.

Proof. Let $x \in X$. Let $y=f(x)$, let $\left(V_{n}\right)_{1}^{\infty}$ be a decreasing local base at $y$ in $Y$, and let $\left(U_{n}\right)_{1}^{\infty}$ be a decreasing local base at $x$ in $f^{-1}(y)$. Choose open $W_{n}$ in $X$ such that $W_{n} \subset f^{-1}\left(V_{n}\right), W_{n} \cap f^{-1}(y) \subset U_{n}$, and $\bar{W}_{n+1} \subset W_{n}$ for all $n$. We claim that $\left(W_{n}\right)_{1}^{\infty}$ is a local base at $x$ in $X$.

Suppose not. Then there is a neighborhood $G$ of $x$ in $X$ such that $W_{n}-G \neq \varnothing$ for all $n$. Pick $x_{n} \in W_{n}-G$. It is easy to see that $\left\{x_{n}: n \in N\right\}$ has no cluster point in $X$, so all its subsets are closed. Choose $n_{0}$ so that $W_{n} \cap f^{-1}(y) \subset G$ for all $n>n_{0}$, and let $A=\left\{x_{n}: n>n_{0}\right\}$. Then $A$ is closed in $X$, but $B=f(A)$ is not closed in $Y$ because $y \in \bar{B}-B$. This contradiction completes the proof.

For our next lemma, whose verification is left to the reader, recall that a space $X$ is a $k$-space if, whenever $A \subset X$ and $A \cap K$ is closed in $K$ for every compact $K \subset X$, then $A$ is closed in $X$.

LEMMA 2.4. If $X$ is a $k$-space, and if every compact $K \subset X$ is a c-space (in particular, metrizable), then $X$ is a c-space.

Lemma 2.5. If $X_{1}$ and $X_{2}$ satisfy (1.5), so does $X_{1} \times X_{2}$.

Proof. Let $\mathscr{P}_{1}$ and $\mathscr{P}_{2}$ be covers of $X_{1}$ and $X_{2}$, respectively, satisfying (1.5). Then $\left\{P_{1} \times P_{2}: P_{1} \in \mathscr{P}_{1}, P_{2} \in \mathscr{P}_{2}\right\}$ is a cover of $X_{1} \times X_{2}$ satisfying (1.5).

\section{Compact spaces.}

THEOREM 3.1. The following properties of a compact Hausdorff space $X$ are equivalent.

(a) $X$ is metrizable.

(b) $X$ satisfies (1.5).

Proof. (a) $\rightarrow$ (b). Obvious.

(b) $\rightarrow$ (a). Let $\mathscr{P}$ be a cover of $X$ as in (1.5); we may assume that $\mathscr{P}$ is closed under finite intersections. Then $\mathscr{P}$ has the following property, which is formally stronger than required by (1.5): If $A \subset X$ is finite and 
$x \in X-A$, then there is a finite $\mathscr{F} \subseteq \mathscr{P}$ such that $x \in(\cup \mathscr{F})^{\circ}$ and $A \cap(\cup \mathscr{F})=\varnothing$.

Let $\mathscr{P}^{\prime}=\cup\{\mathscr{G} \subset \mathscr{P}: \mathscr{G}$ is a minimal finite cover of $X\}$. By Lemma $2.1, \mathscr{P}^{\prime}$ is countable. Let us show that $\mathscr{P}^{\prime}$ also satisfies the condition in (1.5). So suppose $x, y \in X$, with $x \neq y$. Then there is a finite $\mathscr{F} \subset \mathscr{P}$ such that $x \in(\cup \mathscr{F})^{\circ}$ and $y \notin \cup \mathscr{F}$; we may suppose that $x \notin(\cup \mathscr{E})^{\circ}$ if $\mathscr{E} \subset \mathscr{F}$ and $\mathscr{E} \neq \mathscr{F}$. It will suffice to show that $\mathscr{F} \subset \mathscr{P}^{\prime}$. Now for each $F \in \mathscr{F}$, there is some $x_{F} \in F \cap(\cup \mathscr{F})^{\circ}$ such that $x_{F} \notin \cup\left\{F^{\prime} \in \mathscr{F}: F^{\prime} \neq F\right\}$. Let $A=\left\{x_{F}: F \in \mathscr{F}\right\}$. For each $z \in X-A$, there is a finite $\mathscr{F}_{z} \subset \mathscr{P}$ such that $z \in\left(\cup \mathscr{F}_{z}\right)^{\circ}$ and $A \cap\left(\cup \mathscr{F}_{z}\right)=\varnothing$. Define $\mathscr{R} \subset \mathscr{P}$ by

$$
\mathscr{R}=\mathscr{F} \cup\left(\cup\left\{\mathscr{F}_{z}: z \in X-A\right\}\right) .
$$

Then the interiors of finite unions of elements of $\mathscr{R}$ cover $X$, so $\mathscr{R}$ has a finite subcover $\mathscr{G}$ which we may take to be minimal. Clearly $\mathscr{G} \subset \mathscr{P}^{\prime}$. If $F \in \mathscr{F}$, then $F$ is the only element of $\mathscr{R}$ containing $x_{F}$, so $F \in \mathscr{G}$. Hence $\mathscr{F} \subset \mathscr{G} \subset \mathscr{P}^{\prime}$.

We have shown that $\mathscr{P}^{\prime}$ is a countable cover of $X$ satisfying (1.5). Let

$$
\mathscr{B}=\left\{X-(\cup \mathscr{F})^{\circ}: \mathscr{F}_{\mathcal{F}} \subset \mathscr{P}^{\prime}, \mathscr{F} \text { finite }\right\}
$$

Then $\mathscr{B}$ is a countable, closed, point-separating cover of $X$. Since $X$ is compact (countably compact would suffice), the collection of all finite intersections of elements from $\mathscr{B}$ is a countable network for $X$. Thus $X$ has a $G_{\delta}$-diagonal and is therefore metrizable. That completes the proof.

COROllary 3.2. Every Hausdorff $k$-space satisfying (1.5) is a c-space.

Proof. This follows immediately from Lemma 2.4 and Theorem 3.1.

4. Paracompact $M$-spaces. $K$. Morita introduced $M$ spaces in [16, §6], and showed that a space $X$ is a paracompact $M$-space if and only if it admits a perfect map onto a metrizable space $M$. The class of paracompact $M$-spaces coincides with the class of paracompact $p$-spaces defined by A. V. Arhangel'skiǐ [2].

The proof of Theorem 4.2 below depends on Theorem 3.1 and the following result due independently to C. R. Borges [3, Theorem 8.1] and A. Okuyama [19].

THEOREM 4.1 (Borges-Okuyama). A space $X$ is metrizable if and only if it is a paracompact $M$-space and its diagonal $\Delta(X)$ is a $G_{\delta}$ subset of $X \times X$. 
J. Nagata [18] generalized Theorem 4.1 (as well as a result of V. V. Filippov [8, Theorem 3]) by weakening "has a $G_{\delta}$ diagonal" to "satisfies (1.2)". We now generalize Nagata's theorem, as well as Theorem 3.1 above, as follows.

THEOREM 4.2. The following properties of a space $X$ are equivalent.

(a) $X$ is metrizable.

(b) $X$ is a paracompact $M$-space satisfying (1.5).

Proof. (a) $\rightarrow$ (b). Clear.

(b) $\rightarrow$ (a). It will suffice to show that (b) implies that every closed subset of $X$ is a $G_{\delta}$, for we can then apply this result to $X \times X$ to conclude that the diagonal $\Delta(X)$ is a $G_{\delta}$ in $X \times X$, so that $X$ is metrizable by Theorem 4.1. (Note that, if $X$ satisfies (b), then so does $X \times X$, since $X \times X$ is a paracompact $M$-space by [16, Theorem 6.4] and satisfies (1.5) by Lemma 2.5.)

Now assume (b). Since $X$ is a paracompact $M$-space, there is a perfect map $f: X \rightarrow M$ from $X$ onto a metrizable space $M$. Let $\left\{\mathscr{V}_{n}: n \in N\right\}$ be a sequence of locally finite open covers of $M$ such that, for every $y \in M$, the collection $\left\{\operatorname{St}\left(y, \mathscr{V}_{n}\right): n \in N\right\}$ is a local base at $y$ in $M$. Let $\mathscr{U}_{n}=\left\{f^{-1}(V): V \in \mathscr{V}_{n}\right\}$. Then $\left\{\mathscr{U}_{n}: n \in N\right\}$ is a sequence of locally finite open covers of $X$ such that, if $x \in X$ and if $S_{x}$ denotes the compact set $f^{-1}(f(x))$, then $\left\{\operatorname{St}\left(x, \mathscr{U}_{n}\right): n \in N\right\}$ is a neighborhood base about $S_{x}$ in $X$.

It follows from Theorem 3.1 that every compact subset of $X$, and thus in particular every $S_{x}$, is metrizable, so Lemma 2.3 implies that $X$ is first-countable and thus a $c$-space.

Now let $A \subset X$ be closed, and let us show that it is a $G_{\delta}$ in $X$. Let $\mathscr{P}$ be a cover of $X$ satisfying (1.5). For each $U \subset X$, Lemma 2.2 implies that there are at most countably many minimal finite $\mathscr{F} \subset \mathscr{P}$ such that $(U \cap A) \subset(\cup \mathscr{F})^{\circ}$; label these collections $\{\mathscr{F}(U, n): n \in N\}$. (We may assume $X \in \mathscr{P}$, so there is at least one such collection, and we permit $\mathscr{F}(U, n)=\mathscr{F}(U, m)$ for $n \neq m$. $)$ Let

$$
\begin{array}{rr}
U(k)=\bigcap_{j=1}^{k}(U \mathscr{F}(U, j))^{\circ} \cap U & (U \subset X, k \in N), \\
W_{n, k}=U\left\{U(k): U \in \mathscr{U}_{n}\right\} & (n, k \in N) .
\end{array}
$$

Since each $U(k)$ contains $U \cap A$, and $U_{n}$ covers $A$, it is clear that $W_{n, k}$ is an open set about $A$. We will show that $A=\cap\left\{W_{n, k}: n, k \in N\right\}$.

Suppose $x \in X-A$, and let us show that $x \notin W_{n, k}$ for some $n$ and $k$. Since $S_{x} \cap A$ is compact, $x \notin S_{x} \cap A$, and $\mathscr{P}$ satisfies (1.5), there must exist a finite $\mathscr{F} \subset \mathscr{P}$ such that $\left(S_{x} \cap A\right) \subset(\cup \mathscr{F})^{\circ}$ and $x \notin \cup \mathscr{F}$. (If 
$S_{x} \cap A=\varnothing$, take $\left.\mathscr{F}=\varnothing\right)$. Let $G=(X-A) \cup(\cup \mathscr{F})^{\circ}$. Then $G$ is an open set about $S_{x}$, so $\operatorname{St}\left(x, \mathscr{U}_{n}\right) \subset G$ for some $n$. Let $U_{1}, \cdots, U_{s}$ be the elements of the locally finite collection $\mathcal{U}_{n}$ which contain $x$. Then for any $i \leqq s$,

$$
U_{i} \cap A \subset\left(S t\left(x, \mathscr{U}_{n}\right) \cap A\right) \subset(G \cap A) \subset(\cup \mathscr{F})^{\circ},
$$

so we can chose a minimal $\mathscr{F}_{t} \subset \mathscr{F}$ such that $U_{l} \cap A \subset\left(\cup \mathscr{F}_{t}\right)^{\circ}$. Then $\mathscr{F}_{i}=\mathscr{F}\left(U_{i}, k_{\imath}\right)$ for some $k_{i} \in N$. Let $k=\max \left\{k_{i}: i \leqq s\right\}$. Now observe that, if $i \leqq s$, then

$$
U_{l}(k) \subset \cup \mathscr{F}\left(U_{l}, k_{l}\right)=\cup \mathscr{F}_{\imath} \subset \cup \mathscr{F}
$$

Since $x \notin \cup \mathscr{F}$, it follows that $x \notin U(k)$ whenever $U \in \mathcal{U}_{n}$ and $x \in U$. But surely $x \notin U(k)$ if $x \notin U$, so $x \notin U(k)$ for any $U \in U_{n}$. Hence $x \notin W_{n, k}$, and that completes the proof.

It should be remarked that, in general, a paracompact $M$-space whose compact subsets are all metrizable need not itself be metrizable; see [14, Example 5.2].

5. $\sigma$-spaces and $\Sigma$-spaces. A space $X$ is a $\sigma$-space [20] if $X$ has a $\sigma$-locally finite closed network (i.e. a $\sigma$-locally finite cover $\mathscr{A}$ by closed sets such that, if $x \in U$ with $U$ open in $X$, then $x \in A \subset U$ for some $A \in \mathscr{A}$.) A space $X$ is a strong $\Sigma$-space (resp., a $\Sigma$-space) [17] if there is a $\sigma$-locally finite closed cover $\mathscr{A}$ of $X$, and a covering $\mathscr{K}$ of $X$ by compact (resp., countably compact) sets such that, if $K \subset U$ with $K \in \mathscr{K}$ and $U$ open in $X$, than $K \subset A \subset U$ for some $A \in \mathscr{A}$. The class of $\Sigma$-spaces (resp., strong $\Sigma$-spaces) contains all $\sigma$-spaces and all $M$-spaces (resp., paracompact $M$-spaces).

It was proved in [11, Theorem 3] that a regular strong $\Sigma$-space satisfying (1.2) is a $\sigma$-space. We will show in Theorem 5.2 below that (1.2) can be weakened to (1.5) in this result, thereby also enabling us to state our theorem as an equivalence.

To prove Theorem 5.2, (b) $\rightarrow$ (a), we will need a result from [11, 13]. A continuous map $f: X \rightarrow Y$ is called a $\sigma$-locally finite map if every $\sigma$-locally finite cover $\mathscr{A}$ of $X$ has a refinement $\mathscr{B}$ for which $f(\mathscr{B})$ is $\sigma$-locally finite. The following characterization of $\sigma$-spaces and strong $\Sigma$-spaces was given in [11, Theorem 2] and a proof is given in [13, Theorem 1.3].

THEOREM 5.1 [11, 13]. The following properties of a regular space $Y$ are equivalent.

(a) $Y$ is a $\sigma$-space (resp., strong $\Sigma$-space). 
(b) $Y$ is the image under a $\sigma$-locally finite map of a metrizable space (resp., paracompact $M$-space).

Moreover, for (a) $\rightarrow$ (b) the domain can be chosen to be a subset of $Y \times M$ for some metrizable space $M$.

THEOREM 5.2. The following properties of a regular space $Y$ are equivalent.

(a) $Y$ is a $\sigma$-space.

(b) $Y$ is a strong $\Sigma$-space satisfying (1.5).

Proof. (a) $\rightarrow$ (b). (Only need $Y$ to be $T_{1}$.) Suppose $Y$ is a $\sigma$ space. Then $Y$ is clearly a strong $\Sigma$-space, so we need only show that $Y$ satisfies (1.5).

Let $\mathscr{A}=\cup_{n=1}^{\infty} \mathscr{A}_{n}$ be a closed network for $Y$, with each $\mathscr{A}_{n}$ a locally finite cover of $Y$. For all $n$ and all $\mathscr{B} \subset \mathscr{A}_{n}$, let

$$
P_{n}(\mathscr{B})=\cap \mathscr{B}-\cup\left(\mathscr{A}_{n}-\mathscr{B}\right),
$$

let $\mathscr{P}_{n}=\left\{P_{n}(\mathscr{B}): \mathscr{B} \subset \mathscr{A}_{n}\right\}$, and let $\mathscr{P}=\bigcup_{n=1}^{\infty} \mathscr{P}_{n}$. We will show that $\mathscr{P}$ satisfies (1.5).

First, it is easy to check that each $\mathscr{P}_{n}$ is a disjoint cover of $Y$, so $\mathscr{P}$ is surely point-countable. Now suppose $x, y \in Y, x \neq y$. Pick $n$ so that, for some $A_{0} \in \mathscr{A}_{n}, y \in A_{0}$ and $x \notin A_{0}$. For each $z \in Y$, let $\mathscr{B}_{z}=$ $\left\{A \in \mathscr{A}_{n}: z \in A\right\}$, and note that $\mathscr{B}_{z}$ is finite. Let

$$
\mathscr{F}=\left\{P_{n}(\mathscr{B}): \mathscr{B} \subset \mathscr{B}_{x}\right\}
$$

Clearly $\mathscr{F} \subset \mathscr{P}_{n} \subset \mathscr{P}$, and $\mathscr{F}$ is finite. We will show that $y \notin \cup \mathscr{F}$ and $x \in(\cup \mathscr{F})^{\circ}$.

If $\mathscr{B} \subset \mathscr{B}_{x}$, then $A_{0} \in \mathscr{A}_{n}-\mathscr{B}$, so $P_{n}(\mathscr{B}) \subset Y-A_{0}$, and thus $y \notin P_{n}(\mathscr{B})$. Hence $y \notin \cup \mathscr{F}$. To show that $x \in(\cup \mathscr{F})^{\circ}$, let $U=$ $Y-\cup\left(\mathscr{A}_{n}-\mathscr{B}_{x}\right)$; then $x \in U$ and $U$ is open in $Y$, so we need only show that $U=\cup \mathscr{F}$. But if $z \in \cup \mathscr{F}$, then $z \in P_{n}(\mathscr{B})$ for some $\mathscr{B} \subset \mathscr{B}_{x}$, so

$$
z \in P_{n}(\mathscr{B}) \subset Y-\cup\left(\mathscr{A}_{n}-\mathscr{B}\right) \subset Y-\cup\left(\mathscr{A}_{n}-\mathscr{B}_{x}\right)=U
$$

In the other direction, if $z \in U$, then $\mathscr{B}_{z} \subset \mathscr{B}_{x}$, so $P_{n}\left(\mathscr{B}_{z}\right) \in \mathscr{F}$; since $z \in P_{n}\left(\mathscr{B}_{z}\right)$, it follows that $z \in \cup \mathscr{F}$.

(b) $\rightarrow$ (a). Suppose $Y$ is a strong $\Sigma$-space satisfying (1.5). By Theorem 5.1, there exists a metrizable space $M$, a paracompact $M$-space $X \subset Y \times M$, and a $\sigma$-locally finite map $f: X \rightarrow Y$. By Lemma 2.5, $Y \times M$ also satisfies (1.5), and hence so does $X$. Hence $X$ is metrizable by Theorem 4.2 , so $Y$ is a strong $\Sigma$-space by Theorem 5.1. That completes the proof. 
REMARK 5.3. In the proof of Theorem 5.2 , (a) $\rightarrow(b)$, we have actually shown that every space with a $\sigma$-locally finite, point-separating closed cover satisfies (1.5). (See also footnote 4 and Remark 8.3).

REMARK 5.4. We have derived Theorem 5.2, (b) $\rightarrow$ (a), from Theorem 4.2, with the aid of Theorem 5.1. Conversely, Theorem 4.2 follows from Theorem 5.2 and Theorem 4.1, since every paracompact $M$-space is a strong $\Sigma$-space and every Hausdorff $\sigma$-space has a $G_{\delta}$ diagonal.

6. Spaces with point-countable bases. The following result was proved in [6, Theorem 1.2].

THEOREM 6.1 [6]. A space X has a point-countable base if and only if it satisfies (the formally weaker) condition (1.3).

It was shown in [6, Remark 4.4] that Theorem 6.1 becomes false if (1.3) is further weakened to (1.4). We do, however, have the following result.

THEOREM 6.2. The following properties of a regular space $X$ are equivalent.

(a) X has a point-countable base.

(b) $X$ is a $k$-space satisfying (1.4).

(c) $X$ is a c-space satisfying (1.4).

Proof. (a) $\rightarrow$ (b). Clear, since spaces with a point-countable base are first-countable.

(b) $\rightarrow$ (c). By Corollary 3.2.

(c) $\rightarrow$ (a). Let $\mathscr{P}$ be a cover of $X$ satisfying (1.4). Let $\Phi=\{\mathscr{F} \subset \mathscr{P}: \mathscr{F}$ is finite $\}$. For $\mathscr{F} \in \Phi$, let

$$
M(\mathscr{F})=\left\{x \in X: x \in(\cup \mathscr{F})^{\circ}, x \notin(\cup \mathscr{E})^{\circ} \text { if } \mathscr{E} \subset \mathscr{F}, \mathscr{E} \neq \mathscr{F}\right\} .
$$

It follows from Lemma 2.2 that, if $x \in X$, then $x \in M(\mathscr{F})$ for only countably many $\mathscr{F} \in \Phi$. For each $P \in \mathscr{P}$, let

$$
P^{\prime}=P \cup(\cup\{M(\mathscr{F}): \mathscr{F} \in \Phi, P \in \mathscr{F}\})
$$

Then $P^{\prime} \subset \bar{P}$, for if $x \in M(\mathscr{F})$ for some $\mathscr{F} \in \Phi$ with $P \in \mathscr{F}$, then $x \in(\cup \mathscr{F})^{\circ}$ while $x \notin(\cup(\mathscr{F}-\{P\}))^{\circ}$, and thus $x \in \bar{P}$. Now let $\mathscr{P}^{\prime}=\left\{P^{\prime}: P \in \mathscr{P}\right\}$. To show that $X$ has a point-countable base, it will suffice, by Theorem 6.1 , to show that $\mathscr{P}^{\prime}$ satisfies (1.3). 
To see that $\mathscr{P}^{\prime}$ is point-countable, let $x \in X$. Define $\mathscr{A}_{x}=$ $\cup\{\mathscr{F} \in \Phi: x \in M(\mathscr{F})\}$; then $\mathscr{A}_{x}$ is countable by the remark after the definition of $M(\mathscr{F})$. Since $x \in P^{\prime}$ implies that $x \in P$ or $P \in \mathscr{A}_{x}$, it follows that $x \in P^{\prime}$ for only countably many $P \in \mathscr{P}$.

Suppose now that $x \in W$, with $W$ open in $X$. Pick an open $U$ in $X$ such that $x \in U \subset \bar{U} \subset W$. By (1.4), there is an $\mathscr{F} \in \Phi$ such that $x \in$ $(\cup \mathscr{F})^{\circ}$ and $(\cup \mathscr{F}) \subset U$, and we may suppose that $x \in M(\mathscr{F})$. Let $\mathscr{F}^{\prime}=\left\{P^{\prime}: P \in \mathscr{F}\right\}$. We need only show that $x \in\left(\cup \mathscr{F}^{\prime}\right)^{\circ}$ and that $x \in P^{\prime} C$ $W$ for every $P \in \mathscr{F}$.

Since $P^{\prime} \supset P$ for all $P \in \mathscr{P}$, it is clear that $x \in\left(\cup \mathscr{F}^{\prime}\right)^{\circ}$. Since $x \in M(\mathscr{F})$, we have $x \in P^{\prime}$ for every $P \in \mathscr{F}$. Finally, if $P \in \mathscr{F}$, then

$$
P^{\prime} \subset \bar{P} \subset(\cup \mathscr{F})^{-} \subset \bar{U} \subset W,
$$

and that completes the proof.

7. Countably compact spaces. In this section we will slightly generalize the results of $\S \S 3-5$. Our principal tool will be the following lemma.

Lemma 7.1. Suppose $X$ is a countably compact $c$-space, and $\mathscr{P}$ is a point-countable cover of $X$. If $\mathcal{U}$ is an open cover of $X$ consisting of interiors of finite unions from $\mathscr{P}$, then $\mathcal{U}$ has a finite subcover.

Proof. Let $\Phi$ be the family of all finite subcollections $\mathscr{F}$ of $\mathscr{P}$ such that $(\cup \mathscr{F})^{\circ} \subset U$ for some $U \in \mathcal{U}$. For $\mathscr{F} \in \Phi$, let

$$
M(\mathscr{F})=\left\{x \in(\cup \mathscr{F})^{\circ}: x \notin(\cup \mathscr{E})^{\circ} \text { if } \mathscr{E} \subset \mathscr{F}, \mathscr{E} \neq \mathscr{F}\right\},
$$

and note that $(\cup \mathscr{F})^{\circ}=\cup\{M(\mathscr{E}): \mathscr{E} \subset \mathscr{F}\}$. For each $x \in X$, let

$$
\mathscr{V}(x)=\left\{(\cup \mathscr{F})^{\circ}: \mathscr{F} \in \Phi, x \in M(\mathscr{F})\right\}
$$

Clearly $x \in \cup \mathscr{V}(x)$. Since $X$ is a $c$-space, it follows from Lemma 2.2 that $\mathscr{V}(x)$ is countable.

Suppose now that $\mathcal{U}$ did not have a finite subcover. Then the union of finitely many of the $\mathscr{V}(x)$ cannot cover $X$, for if it did, $X$ (being countably compact) would be covered by finitely many $(\cup \mathscr{F})^{\circ}$ with $\mathscr{F} \in \Phi$, and hence also by finitely many $U \in \mathcal{U}$. By induction, we can therefore pick a sequence $x_{n} \in X$ such that $x_{n} \notin \cup\left(\mathscr{V}\left(x_{m}\right)\right)$ if $m<n$. These $x_{n}$ are distinct, and the sequence $\left\{x_{n}\right\}_{1}^{\infty}$ has a cluster point $x \in X$. Since $\mathcal{U}$ covers $X$, there is an $\mathscr{F} \in \Phi$ such that $x \in(\cup \mathscr{F})^{\circ}$. Then $(\cup \mathscr{F})^{\circ}$ contains infinitely many $x_{k}$. Also, since $(\cup \mathscr{F})^{\circ}=\cup\{M(\mathscr{E}): \mathscr{E} \subset \mathscr{F}\}$ and there are only finitely many $\mathscr{E} \subset \mathscr{F}$, there must be an $\mathscr{E} \subset \mathscr{F}$ such that 
$M(\mathscr{E})$ contains infinitely many $x_{k}$ (even though $x$ may not be an element of $M(\mathscr{E}))$. Pick $m, n \in N$ with $m<n$ such that $x_{m}, x_{n} \in M(\mathscr{E})$. Then $(\cup \mathscr{E})^{\circ} \in \mathscr{V}\left(x_{m}\right)$, so

$$
x_{n} \in M(\mathscr{E}) \subset(\cup \mathscr{E})^{\circ} \subset \cup\left(\mathscr{V}\left(x_{m}\right)\right)
$$

Since $m<n$, this contradicts the choice of $x_{n}$, and that completes the proof.

Lemma 7.1 should be compared to the following result of G. Aquaro [1]. Every point-countable open cover of a countably compact space $X$ has a finite subcover. One need not assume, in Aquaro's result, that $X$ is a $c$-space, and we do not know whether that assumption is really necessary in Lemma 7.1. If it can be omitted from Lemma 7.1, then one can also omit the requirement that $X$ is a $c$-space or a $k$-space from Theorems 7.2-7.4 below. It should be remarked, that, by Aquaro's result, Theorems 7.2-7.4 are valid without this requirement if (1.5) is replaced by the stronger condition (1.2).

Our next result generalizes Theorem 3.1.

THEOREM 7.2. Suppose $X$ is a countably compact Hausdorff space which is a $c$-space or a $k$-space. Then the following are equivalent.

(a) $X$ is metrizable (and thus compact).

(b) $X$ satisfies (1.5).

Proof. (a) $\rightarrow$ (b). Clear.

(b) $\rightarrow(a)$. If $X$ is a $k$-space, then it is a $c$-space by Corollary 3.2. We may therefore assume that $X$ is a $c$-space. Observe now that the whole proof of Theorem 3.1, (b) $\rightarrow$ (a), remains valid under our hypothesis, with two modifications: That the cover $\mathscr{R}$ has a finite subcover is obvious in a compact space; in a countably compact $c$-space, it follows from Lemma 7.1. Then, after the existence of a countable network for $X$ is established, it follows that $X$ is Lindelöf and therefore compact. That completes the proof.

Theorem 7.2 implies that a Hausdorff $M$-space (resp., $\Sigma$-space) which satisfies (1.5), and which is either a $c$-space or a $k$-space, is a paracompact $M$-space (resp., a strong $\Sigma$-space). We therefore obtain the following results.

THEOREM 7.3. Theorem 4.2 remains true with "paracompact $M$ space" weakened to "Hausdorff $M$-space which is a $c$-space or a $k$-space". 
THEOREM 7.4. Theorem 5.3, (b) $\rightarrow$ (a), remains true with "strong $\Sigma$-space" changed to " $\Sigma$-space which is a c-space or a $k$-space".

8. Modifications of property (1.5). Consider the following natural modifications of property (1.5).

$(1.5)^{-}$. $X$ has a point-countable cover $\mathscr{P}$ such that if $x, y \in X$ with $x \neq y$, there is a finite subcollection $\mathscr{F}$ of $\mathscr{P}$ such that $x \in(\cup \mathscr{F})^{\circ}$ and $y \notin(\cup \mathscr{F})^{\circ}$.

$(1.5)^{+} . \quad X$ has a point-countable cover $\mathscr{P}$ such that if $x, y \in X$ with $x \neq y$, there is a finite subcollection $\mathscr{F}$ of $\mathscr{P}$ such that $x \in(\cup \mathscr{F})^{\circ}=\cup \mathscr{F}$ and $y \notin(\cup \mathscr{F})$.

It is clear that $(1.5)^{-}$is formally weaker than $(1.5)$, and $(1.5)^{+}$is formally stronger than (1.5). The next theorem shows that, in $c$-spaces, $(1.5)^{-},(1.5)$, and $(1.5)^{+}$are equivalent.

THEOREM 8.1. For any $c$-space $X$ the following are equivalent.

(a) $X$ satisfies $(1.5)^{-}$.

(b) $X$ satisfies (1.5).

(c) $X$ satisfies $(1.5)^{+}$.

Proof. (c) $\rightarrow$ (b) $\rightarrow$ (a). Trivial.

$($ a) $\rightarrow$ (c). Let $\mathscr{P}$ be a point-countable cover of $X$ as given in $(1.5)^{-}$. Let $\Phi$ be the collection of all finite subcollections of $\mathscr{P}$. For $\mathscr{F} \in \Phi$, let

$$
M(\mathscr{F})=\left\{x \in(\cup \mathscr{F})^{\circ}: x \notin(\cup \mathscr{E})^{\circ} \text { if } \mathscr{E} \subset \mathscr{F}, \mathscr{E} \neq \mathscr{F}\right\}
$$

and note that $(\cup \mathscr{F})^{\circ}=\cup\{M(\mathscr{E}): \mathscr{E} \subset \mathscr{F}\}$. If $\mathscr{P}^{\prime}=\{M(\mathscr{F}): \mathscr{F} \in \Phi\}$, it follows from Lemma 2.2 that every $x \in X$ is in at most countably many elements of $\mathscr{P}^{\prime}$, and therefore $\mathscr{P}^{\prime}$ is point-countable. To show that $\mathscr{P}^{\prime}$ satisfies $(1.5)^{+}$, let $x, y \in X$ with $x \neq y$; then there is some $\mathscr{F} \in \Phi$ such that $x \in(\cup \mathscr{F})^{\circ}$ and $y \notin(\cup \mathscr{F})^{\circ}$. Let $\mathscr{F}^{\prime}=\{M(\mathscr{E}): \mathscr{E} \subset \mathscr{F}\}$. Then $\mathscr{F}^{\prime}$ is a finite subcollection of $\mathscr{P}^{\prime}$, and

$$
\cup \mathscr{F}^{\prime}=\cup\{M(\mathscr{E}): \mathscr{E} \subset \mathscr{F}\}=(\cup \mathscr{F})^{\circ} .
$$

Hence $x \in\left(\cup \mathscr{F}^{\prime}\right)^{\circ}=\cup \mathscr{F}^{\prime}$, and $y \notin(\cup \mathscr{F})^{\circ}=\cup \mathscr{F}^{\prime}$. That completes the proof.

The authors do not know whether Theorem 8.1 remains true without the $c$-space assumption, or even whether (1.5) can be weakened to $(1.5)^{-}$ in Theorems 3.1 and 4.2. We do, however, have the following result, which follows immediately from Theorem 8.1 and Corollary 3.2. 
COROllary 8.2. In any Hausdorff $k$-space, properties (1.5) and $(1.5)^{+}$are equivalent.

The following remark follows from the proof of Theorem 5.2, (a) $\rightarrow$ (b).

REMARK 8.3. Every space with a $\sigma$-locally finite, point-separating closed cover satisfies $(1.5)^{+}$.

As mentioned earlier, one of the reasons for showing the equivalence $(1.1) \leftrightarrow(1.3)$ is to provide a simple proof that property (1.1) (and hence (1.3)) is preserved under a perfect map (more generally, a bi-quotient $s$-map). The authors do not know if condition (1.2) is preserved under a perfect map, but we have the following result for (1.5) and $(1.5)^{+}$.

THEOREM 8.4. Let $f: X \rightarrow Y$ be a perfect map. If $X$ satisfies (1.5) (resp., $(1.5)^{+}$), then so does $Y$.

Proof. We prove the theorem for property (1.5); the proof for $(1.5)^{+}$ is similar.

Assume $\mathscr{P}$ is a point-countable cover of $X$ as given in (1.5). Let $\Phi$ be the family of all finite subcollections of $\mathscr{P}$. For $\mathscr{F} \in \Phi$, let

$$
M(\mathscr{F})=\left\{y \in Y: \mathscr{F} \text { is a minimal cover of } f^{-1}(y)\right\},
$$

and let $\mathscr{P}^{\prime}=\{M(\mathscr{F}): \mathscr{F} \in \Phi\}$. It follows from Lemma 2.1 that $\mathscr{P}^{\prime}$ is a point-countable collection of subsets of $Y$; let us show that $\mathscr{P}^{\prime}$ satisfies (1.5). If $z, y \in Y$ with $z \neq y$, pick a fixed point $x \in f^{-1}(y)$. Using the compactness of $f^{-1}(z)$ and property (1.5), we can find an $\mathscr{F} \in \Phi$ such that $f^{-1}(z) \subset(\cup \mathscr{F})^{\circ}$ and $x \notin \cup \mathscr{F}$. Let $\mathscr{F}^{\prime}=\{M(\mathscr{E}): \mathscr{E} \subset \mathscr{F}\}$; then $\mathscr{F}^{\prime}$ is a finite subcollection of $\mathscr{P}$ and $\cup \mathscr{F}^{\prime}=\left\{u \in Y: f^{-1}(u) \subseteq \cup \mathscr{F}\right\}$. Hence $y \notin \cup \mathscr{F}^{\prime}$. If $W=Y-f\left(X-(\cup \mathscr{F})^{\circ}\right)$, then $W$ is open in $Y, z \in W$ and $W \subset \cup \mathscr{F}^{\prime}$. Thus $z \in\left(\cup \mathscr{F}^{\prime}\right)^{\circ}$, and the theorem is proved.

Proposition 8.5. If $X$ is a c-space satisfying $(1.5)^{-}$, then every $x \in X$ is $a G_{\delta}$ in $X$.

Proof. Let $\mathscr{P}$ be as in (1.5)- For each $x \in X$, let $\Phi_{x}$ be the family of all minimal finite $\mathscr{F} \subset \mathscr{P}$ such that $x \in(\cup \mathscr{F})^{\circ}$. By Lemma 2.2, $\Phi_{x}$ is countable. But $(1.5)^{-}$implies that $\cap\left\{(\cup \mathscr{F})^{\circ}: \mathscr{F} \in \Phi_{x}\right\}=\{x\}$, and that completes the proof.

Remark 8.6. Proposition 8.5 and Corollary 3.2 can be combined 
with Theorem 7.3 of [12] to obtain various analogues of Theorem 4.2. (For example, one can conclude that a Hausdorff space satisfying (1.5) is of pointwise countable type if and only if it is first-countable).

\section{REFERENCES}

1. G. Aquaro, Point countable open coverings in countably compact spaces, General Topology and its Relations to Modern Analysis and Algebra, II, Academic Press (1967).

2. A. V. Arhangel'skiǐ, On a class of spaces containing all metric and all locally bicompact spaces, Dokl. Akad. Nauk SSSR, 151 (1963), 751-754. (= Soviet Math. Dokl., 4 (1963), 1051-1055).

3. C. R. Borges, On stratifiable spaces, Pacific J. Math., 17 (1966), 1-16.

4. D. Burke, On point-countable separating open covers in Moore spaces, Notices Amer. Math. Soc., 22 (1975), Abstract 75T-G34, p. A-334.

5. D. Burke and D. Lutzer, Recent advances in the theory of generalized metric spaces, to appear in the Proceedings of the Memphis State Topology Conference, 1975.

6. D. Burke and E. Michael, On a theorem of V. V. Filippov, Israel J. Math., 11 (1972), 394-397.

7. H. H. Corson and E. Michael, Metrizability of certain countable unions, Illinois J. Math., 8 (1964), $351-360$.

8. V. V. Filippov, On feathered paracompacta, Dokl. Akad. Nauk SSSR, 183 (1968), 555-558. (= Soviet Math. Dokl., 9 (1968), 161-164).

9. ㄴ Q Quotient spaces and multiplicity of a base, Mat. Sb., 80 (1969), 521-532. (= Math. USSR - Sb., 9 (1969), 487-496).

10. S. Hanai, On open mappings. II, Proc. Japan Acad., 37 (1961), 233-238.

11. E. Michael, On Nagami's $\Sigma$-spaces and some related matters, Proc. Washington State Univ. Conference on General Topology (1970), 13-19.

12. - A quintuple quotient quest, General Topology and Appl. 2 (1972), 91-138.

13. - $\sigma$-locally finite maps, Proc. Amer. Math. Soc., (to appear).

14. E. Michael and K. Nagami, Compact-covering images of metric spaces, Proc. Amer. Math. Soc., 37 (1973), 260-266.

15. A. S. Miščenko, Spaces with a pointwise denumerable basis, Dokl. Akad. Nauk SSSR, 145 (1962), 985-988. ( = Soviet Math. Dokl., 3 (1962), 855-858).

16. K. Morita, Products of normal spaces with metric spaces, Math. Ann., 154 (1964), 365-382.

17. K. Nagami, ¿-spaces, Fund. Math., 65 (1969), 169-192.

18. J. Nagata, A note on Filipov's theorem, Proc. Japan Acad., 45 (1969), 30-33.

19. A. Okuyama, On metrizability of $M$-spaces, Proc. Japan Acad., 40 (1964), 176-179.

20. - $-\sigma$-spaces and closed mappings, Proc. Japan Acad., 44 (1968), 472-477.

21. V. I. Ponomarev, Axioms of countability and continuous mappings, Bull. Acad. Polon. Sci. Sér. Math. Astronom. Phys., 8 (1960), 127-134. (Russian).

22. T. Shiraki, M-spaces, their generalizations and metrization theorems, Sci. Rep. Tokyo Kyoiku Daigaku Sect. A, 11 (1971), 57-67.

Received November 24, 1975.

MiAMI UNIVERSITY

AND

UNIVERSITY OF WASHINGTON 



\section{Pacific Journal of Mathematics}

Vol. 64, No. 1

May, 1976

Walter Allegretto, Nonoscillation theory of elliptic equations of order $2 n \ldots \ldots \quad 1$

Bruce Allem Anderson, Sequencings and starters.................. 17

Friedrich-Wilhelm Bauer, A shape theory with singular homology .......... 25

John Kelly Beem, Characterizing Finsler spaces which are

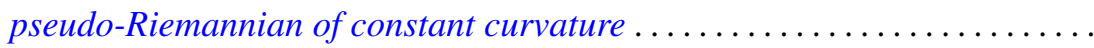

Dennis K. Burke and Ernest A. Michael, On certain point-countable

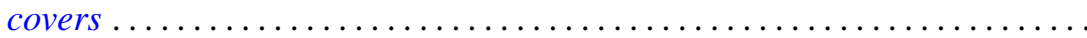

Robert Chen, A generalization of a theorem of Chacon ............... 93

Francis H. Clarke, On the inverse function theorem ................ 97

James Bryan Collier, The dual of a space with the Radon-Nikodým

property ....................................... 103

John E. Cruthirds, Infinite Galois theory for commutative rings ............ 107

Artatrana Dash, Joint essential spectra......................... 119

Robert M. DeVos, Subsequences and rearrangements of sequences in FK

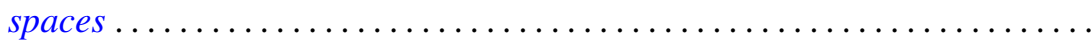

Geoffrey Fox and Pedro Morales, Non-Hausdorff multifunction generalization

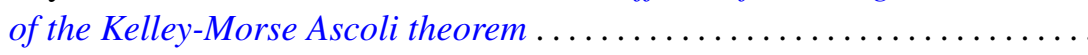

Richard Joseph Fleming, Jerome A. Goldstein and James E. Jamison, One

parameter groups of isometries on certain Banach spaces.............

Robert David Gulliver, II, Finiteness of the ramified set for branched

immersions of surfaces

Kenneth Hardy and István Juhász, Normality and the weak cb property ...... 167

C. A. Hayes, Derivation of the integrals of $L^{(q)}$-functions.

Frederic Timothy Howard, Roots of the Euler polynomials .

Robert Edward Jamison, II, Richard O'Brien and Peter Drummond Taylor, On

embedding a compact convex set into a locally convex topological vector space ....................................

Andrew Lelek, An example of a simple triod with surjective span smaller than span ...

Janet E. Mills, Certain congruences on orthodox semigroups

Donald J. Newman and A. R. Reddy, Rational approximation of $e^{-x}$ on the positive real axis.

John Robert Quine, Jr., Homotopies and intersection sequences ...

Nambury Sitarama Raju, Periodic Jacobi-Perron algorithms and fundamental units ....

Herbert Silverman, Convexity theorems for subclasses of univalent functions. . .

Charles Frederick Wells, Centralizers of transitive semigroup actions and endomorphisms of trees.........................

Volker Wrobel, Spectral approximation theorems in locally convex spaces ..................... 